\title{
The extent to which standardized uptake values reflect FDG phosphorylation in the liver and spleen as functions of time after injection of ${ }^{18}$ F-fluorodeoxyglucose
}

\author{
Georgia Keramida', Constantinos D. Anagnostopoulos ${ }^{2}$ and A. Michael Peters ${ }^{1,3^{*}}$
}

\begin{abstract}
Purpose: In FDG PET/CT, standardized uptake value (SUV) is used to measure metabolic activity but detects un-phosphorylated FDG as well as phosphorylated FDG (FDG6P). Our aim was to determine the proportions of intrahepatic and intrasplenic FDG that are phosphorylated after FDG injection and compare them with SUVs.

Methods: Sixty patients undergoing whole-body PET/CT 60 min post-injection of FDG first had dynamic PET imaging for 30 min with measurement of hepatic and splenic FDG clearances using Patlak-Rutland analysis. The gradient of the Patlak-Rutland plot, which is proportional to clearance (Ki), was normalized to the intercept, which is proportional to FDG distribution volume $(V(0))$ with the same proportionality constant. Using measured values of $\mathrm{Ki} / V(0)$, FDG6P/FDG ratios as functions of time in the two organs were measured for assumed FDG blood disappearance half-times of 40, 50 and $60 \mathrm{~min}$. Hepatic and splenic SUVs were measured from whole-body PET/CT.

Results: The mean (SD) Ki/ $V(0)$ was $0.0036(0.0021)$ and $0.0060(0.0041) \mathrm{ml} / \mathrm{min} / \mathrm{ml}$ for the liver and spleen, respectively, but the hepatic SUV was 1.36 -fold higher than the splenic SUV. This discrepancy was explained by the hepatic $V(0)$ being 1.6-fold higher than the splenic $V(0)$. The percentages of FDG phosphorylated 60 min post-injection were 27, 25 and 23\% for the liver and 39, 36 and 34\% for the spleen, for blood clearance half-times of 40, 50 and $60 \mathrm{~min}$, respectively. SUV indices correlated poorly with $\mathrm{Ki} / \mathrm{V}(0)$ for both organs.
\end{abstract}

Conclusions: SUV is largely determined by un-phosphorylated FDG in dynamic exchange with blood FDG, explaining the poor correlations between SUV indices and $\mathrm{Ki} / V(0)$.

Keywords: FDG clearance, SUV, Liver, Spleen

\section{Background}

Positron emission tomography (PET) with 2-deoxy-2$\left[{ }^{18} \mathrm{~F}\right]$ fluoro-D-glucose (FDG) is widely used to measure tissue glucose utilization rate (MRglu). MRglu is the generation rate of glucose-6-phosphate via glucokinasemediated phosphorylation. FDG, which is an analogue of glucose, also undergoes glucokinase-mediated phosphorylation to FDG-6-phosphate (FDG6P) (Fig. 1). Taking into account a 'lumped constant', introduced to account for

\footnotetext{
* Correspondence: a.m.peters@bsms.ac.uk

${ }^{1}$ Clinical Imaging Sciences Centre, Brighton Sussex Medical School, Brighton, UK ${ }^{3}$ Department of Nuclear Medicine, Royal Sussex County Hospital, Eastern Road, Brighton BN2 5BE, UK

Full list of author information is available at the end of the article
}

differing tissue uptake kinetics between glucose and FDG (for example differing extraction efficiencies), MRglu $(\mu \mathrm{mol} / \mathrm{min} / \mathrm{ml})$ is measured as the tissue FDG clearance rate $(\mathrm{ml} / \mathrm{min} / \mathrm{ml})$ multiplied by blood glucose $(\mu \mathrm{mol} / \mathrm{ml})$. Tissue FDG uptake, however, is often quantified as the standardized uptake value (SUV) recorded on whole-body imaging, conventionally $60 \mathrm{~min}$ post-injection of FDG. SUV is defined as follows:

$$
\mathrm{SUV}=F / \mathrm{ml} \times \text { whole body metric }
$$

where $F / \mathrm{ml}$ is the fraction of administered activity per $\mathrm{ml}$ of tissue; the whole-body metric may be weight (SUW), lean body mass (LBM; SUL) or body surface area. Moreover, SUV may be based on the voxel with 


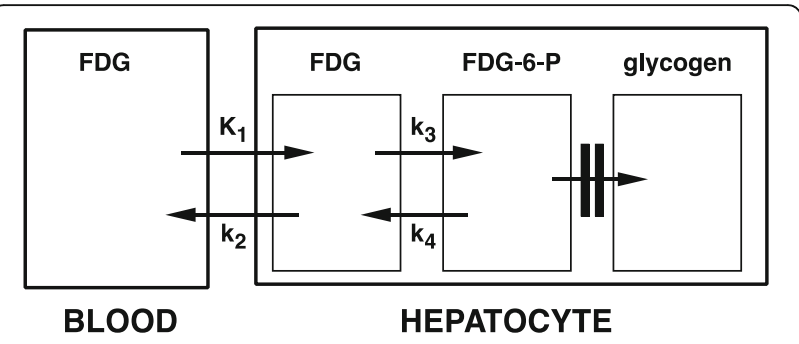

Fig. 1 Model of intrahepatic FDG kinetics. $K_{1}$ is hepatic blood flow, $k_{2}$ is a diffusion constant, $k_{3}$ the glucokinase and $k_{4}$ glucose-6-phosphatase. FDG is assumed to mix throughout its intrahepatic distribution volume via $K_{1}$ and $k_{2}$ by $2-3$ min post-injection. De-phosphorylation (via $k_{4}$ ) is assumed to be slow enough to ignore in Patlak-Rutland analysis, which therefore measures phosphorylation rate. The corresponding model for the spleen is similar except $k_{4}$ is lacking

the maximum value $\left(\mathrm{SUV}_{\max }\right)$ or taken as the mean of all voxels in the region of interest (ROI; $\left.\mathrm{SUV}_{\text {mean }}\right)$.

In addition to the amount of FDG6P in a tissue, SUV also depends on the amount of un-phosphorylated FDG, which is free to exchange between tissue and blood [1-4].

Like FDG clearance, SUV is inversely related to blood glucose in insulin-insensitive tissues such as tumours [5] and brain [6,7]. SUV is therefore a surrogate of FDG clearance rather than of MRglu and becomes a closer surrogate of MRglu after normalization to (i.e. multiplication by) blood glucose, as recommended in the European Association of Nuclear Medicine guidelines on PET/CT [8].

There have been several recent studies of FDG accumulation in the liver, mostly in relation to hepatic steatosis, that have used SUV as the quantitative index of FDG accumulation and measure of hepatic metabolic activity [9-13]. However, these studies have largely ignored the issue of how much intrahepatic FDG is actually phosphorylated. The same applies to the spleen, the metabolic activity of which, almost exclusively based on SUV in previous PET studies, has attracted a great deal of recent attention in relation to atherosclerosis [14] and acute myocardial infarction $[15,16]$, the so-called cardio-splenic axis. Using a combined theoretical and experimental approach, the aim of this study was to determine the proportions of FDG in the liver and spleen that are phosphorylated at specific post-injection times and the extent to which SUV reflects FDG clearance in these two organs.

\section{Methods}

\section{Theory}

The concentration of FDG6P in tissue at time $t$ postinjection can be calculated from the tissue FDG clearance (Ki) and the area under the blood time-concentration curve up to time $t(A(t))$ as follows.

$$
\begin{aligned}
& \mathrm{FDG6P}=A(t) \times \mathrm{Ki} \\
& (\mathrm{MBq} / \mathrm{ml}=[\mathrm{MBq} / \mathrm{ml} \times \mathrm{min}] \times \mathrm{ml} / \mathrm{min} / \mathrm{ml}
\end{aligned}
$$

The concentration of un-phosphorylated FDG (FDG) in tissue at time $t$ post-injection can be calculated from the blood FDG concentration at time $t(C(t))$,

$$
\begin{aligned}
& \mathrm{FDG}=C(t) \times V(0) \\
& (\mathrm{MBq} / \mathrm{ml}=\mathrm{MBq} / \mathrm{ml} \times \mathrm{ml} / \mathrm{ml})
\end{aligned}
$$

where $V(0)$ is the volume of distribution of FDG in the tissue (i.e. the virtual volume within which FDG would be distributed to give an FDG concentration identical to plasma concentration). (If hepatic FDG concentration was identical to plasma concentration, then $V(0)$ would be $1 \mathrm{ml} / \mathrm{ml}$.)

Dividing Eq. 2 by Eq. 3

$$
\mathrm{FDG6P} / \mathrm{FDG}=[A(t) / C(t)] \times[\mathrm{Ki} / V(0)]
$$

Note that $A(t) / C(t)$ is normalised time in a PatlakRutland plot.

There is a very extensive literature on FDG arterial blood clearance data $[1,2,17-28]$, but most publications aimed to validate simplifications of arterial input and very few to quantify the kinetics of blood FDG clearance. From an inspection of published data, the clearance curve can be seen to be a triple exponential with a very fast first exponential that is completed within seconds and a second exponential that is fast with a half-time of $<5 \mathrm{~min}$. The area enclosed by these early exponentials is small compared to the area under the slowest exponential and can be ignored. Three groups in particular have published clearance data in large patient numbers, and from their illustrations, the half-times of the slow exponentials can be seen to be about $40 \mathrm{~min}$ [23], $50 \min$ [19] or $60 \min [21,22,25]$.

Ignoring the fast exponentials, the area under the blood clearance curve is given as

$$
\left.A(t)=(N / \beta) \cdot\left(1-\mathrm{e}^{-\beta . t}\right)\right]
$$

where $N$ is the zero time intercept and $\beta$ the rate constant of the slow exponential,

and

$$
C(t)=N \cdot \mathrm{e}^{-\beta \cdot t}
$$

Therefore,

$$
A(t) / C(t)=\left(1-\mathrm{e}^{-\beta . t}\right) / \beta . e^{-\beta . t}
$$

For blood disappearance half-times of 40, 50 and $60 \mathrm{~min}, A(t) / C(t)$ is 105,94 and $87 \mathrm{~min}$, respectively.

The total tissue concentration of FDG (FDG6P + FDG), which determines SUV, is given by Eqs. 2 and 3: 


$$
\begin{aligned}
\mathrm{FDG} 6 \mathrm{P}+\mathrm{FDG}= & {[A(t) \cdot \mathrm{Ki}]+[C(t) \cdot V(0)] } \\
\mathrm{FDG6P}+\mathrm{FDG}= & (V(0) \cdot A(t) \cdot[\mathrm{Ki} / V(0)]) \\
& +[C(t) \cdot V(0)]
\end{aligned}
$$

It can be seen from Eq. 9 that the relationship between the total activity and $\mathrm{Ki} / V(0)$ has a gradient of $V(0) \cdot A(t)$ and an intercept of $V(0) . C(t)$.

Combining Eqs. 5, 6 and 9

$$
\begin{aligned}
\mathrm{FDG6P}+\mathrm{FDG}= & {\left[V(0) \cdot(N / \beta) \cdot\left(1-\mathrm{e}^{-\beta \cdot t}\right)\right] \cdot \mathrm{Ki} / V(0) } \\
& +\left[V(0) \cdot N \cdot \mathrm{e}^{-\beta \cdot t}\right]
\end{aligned}
$$

When $t=$ infinity, it can be seen from Eq. 10 that

$$
\mathrm{FDG6P}+\mathrm{FDG}=V(0) \cdot(N / \beta) \cdot(\mathrm{Ki} / V(0))
$$

Therefore, when

$$
\begin{aligned}
& \mathrm{Ki} / V(0)=\beta \\
& \mathrm{FDG6P}+\mathrm{FDG}=V(0) \cdot N
\end{aligned}
$$

That is, the total activity remains constant as a function of time following injection when $\mathrm{Ki} / V(0)=\beta$.

\section{Patients}

Sixty patients had dynamic PET prior to routine clinically indicated PET/CT. These patients formed the study group for a previous study that investigated the relationships of hepatic MRglu with hepatic steatosis and obesity [29]. Thirty-eight (including 12 with metabolically active lymphoma) had FDG-avid malignancy on routine PET/ CT. Forty-seven were men (age range 28-84) and 13 were women (age 40-67). Nineteen patients had hepatic steatosis (CT density $\leq 40 \mathrm{HU}$ on whole-body PET/CT), 18 were obese (BMI $\geq 30 \mathrm{~kg} / \mathrm{m}^{2}$ ) and 5 had type 2 diabetes mellitus (none with type 1). Five patients had blood glucose $>7 \mathrm{mmol} / \mathrm{l}$, including one with $>10 \mathrm{mmol} / \mathrm{ml}$ (three of these five were diabetic). Twelve patients had received chemotherapy within 6 months of their scan, 19 had received chemotherapy $>6$ months previously and 29 were chemotherapy-naïve. Patients with known or suspected high ethanol intake were excluded. All patients gave written informed consent for the study, which was approved by a local institutional review board (NRES Committee South Central - Oxford C: ref 13/SC/0231).

\section{Whole-body imaging and image analysis}

Patients fasted for $6 \mathrm{~h}$ before FDG injection. Blood glucose was measured using a glucometer (ACCU-CHEK Performa; Inform ll strips; USA). Whole-body PET/CT was performed at $60 \mathrm{~min}$ post-injection of $\sim 400 \mathrm{MBq}$ FDG (not adjusted for body weight) using a Siemens Biograph 64-slice PET/CT scanner (Erlangen, Germany) with immediate non-enhanced CT scanning (120 Kvp/ $50 \mathrm{~mA}-$ Care dose 4D; slice $5 \mathrm{~mm}$; pitch 0.8 ; rotational speed $0.5 / \mathrm{s}$ ) for attenuation purposes only. 3D emission data was then acquired at 3 min per bed position (PET reconstruction: 4 iterations; subset 8; Gaussian pre-filter; FWHM 5 mm; matrix size $168 \times 168$; zoom 1 ).

Hepatic SUV indices and CT density were measured from a circular ROI of 3-cm diameter over the right lobe of the liver, and spleen SUV indices from a circular ROI of $2.5-\mathrm{cm}$ diameter over the centre of the organ. Body weight and LBM, estimated from height and weight using the equations of Boer [30], were used as the whole-body metrics to give SUW and SUL, respectively. Both were expressed as the maximum voxel SUV ( $\mathrm{SUW}_{\max }$ and $\mathrm{SUL}_{\max }$ ) and mean SUV in the ROI (SUW mean and $\left.\mathrm{SUL}_{\text {mean }}\right)$. We assume that because it is soluble in water rather than fat, negligible FDG enters the fat droplets in hepatocytes. Using an equation relating fat content to CT density [31], hepatic SUV was therefore adjusted for the physical 'dilutional' effect of hepatic fat on the FDG signal, as previously described [32].

\section{Dynamic imaging and image analysis}

Prior to whole-body imaging, dynamic imaging was performed as $30 \times 1$-min frames following FDG injection with detectors positioned over the torso. Hepatic and splenic FDG clearances were measured using PatlakRutland graphical analysis from ROI over the liver $(3 \mathrm{~cm})$ and spleen $(2.5 \mathrm{~cm})$, each summed from 20 contiguous cranio-caudal transaxial slices, avoiding any suspected focal pathology in each slice, as previously described [29]. All frames were corrected for physical decay of ${ }^{18} \mathrm{~F}$. Input function was derived from ROI over the abdominal aorta, within and avoiding the walls, summed from about 20 contiguous cranio-caudal transaxial slices drawn by a single operator (GK). The gradient of the plot, which represents tissue FDG clearance (Ki), was divided by the intercept, which represents tissue distribution volume of FDG (V(0)-see Eq. 3). There was no need for attenuation correction of the dynamic study because the factors that respectively relate gradient and intercept based on raw count rates to Ki and $V(0)$ are identical, and cancel out in their ratio, as previously proven [29].

We did not measure the transport constants governing tissue FDG kinetics shown in Fig. 1. Previous workers have shown that $K_{1}$ (which represents tissue blood flow) and $k_{2}$ (the constant of diffusion of un-phosphorylated FDG from tissue to blood) are sufficiently high with respect to the liver that FDG mixes throughout its hepatic distribution volume within $2-3 \mathrm{~min}$. The first two frames of the dynamic study (0-1 min and 1-2 min) over the liver were therefore excluded from the PatlakRutland plot to allow mixing of the FDG throughout its hepatic distribution volume. The validity of this is 
apparent from Fig. 2, which shows the Patlak-Rutland plot to be essentially linear from the third frame. Although the corresponding transport constants for the spleen have not, to our knowledge, been determined, the plots for the spleen were also linear from the third frame, suggesting that the mixing time of FDG in splenic $V(0)$ is as rapid as in liver $V(0)$. Linearity of the plots also suggests that de-phosphorylation of FDG6P is very slow. Otherwise, the plot would be convex upwards. Dephosporylation takes place via glucose-6-phosphatase $\left(k_{4}\right)$, which is absent from the spleen. The essential requirement of the Patlak-Rutland plot for a single transport constant is therefore met for both tissues when the plot is started from $3 \mathrm{~min}$.

The ratio of FDG6P to total tissue FDG concentration (FDG6P/[FDG6P + FDG]) was calculated from Eqs. 4 and 7 using $A(t) / C(t)$ based on slow exponential halftimes of 40,50 and $60 \mathrm{~min}$ (corresponding to $\beta$ of 0.0173, 0.0139 and $0.0115 \mathrm{~min}^{-1}$, respectively). Total ${ }^{18} \mathrm{~F}$ tracer concentrations were estimated using Eq. 9. $C(t)$ and $V(0)$ are unknown in Eq. 9, so the absolute concentration of tracer is unknown. However, the time courses of tissue FDG6P + FDG concentration for different values of $\mathrm{Ki} / \mathrm{V}(0)$ can be derived because $V(0)$ is independent of post-injection time.

\section{Statistics}

Using the Shapiro-Wilk test, the $W$ statistic gave $p>0.05$ for all SUV indices and $\mathrm{Ki} / V(0)$, so parametric statistics were used. Values were expressed as mean \pm standard deviation (SD). Pearson's correlation analysis was used to assess relationships between SUV indices and $\mathrm{Ki} / V(0)$.

\section{Results}

$\mathrm{Ki} / V(0)$

Representative examples of hepatic and splenic PatlakRutland plots from the same patient are shown in Fig. 2. Both Patlak-Rutland plots are essentially linear from the third frame. For all 60 patients, the mean (SD) Ki/V(0) of the liver was $0.0036(0.0021)$ and of the spleen was $0.0060(0.0041) \mathrm{ml} / \mathrm{min} / \mathrm{ml}(p<0.0001)$.

\section{Estimations of phosphorylated (FDG6P) and un-phosphorylated FDG}

Using the above mean values of $\mathrm{Ki} / V(0)$, the percentages of FDG phosphorylated 60 min post-injection for blood clearance half-times of 40,50 and $60 \mathrm{~min}$ were calculated to be 28,25 , and $23 \%$ for the liver and 39,36 and $34 \%$ for the spleen (Fig. 3). In either organ, percentages approaching $100 \%$ would not be achieved until about $4 \mathrm{~h}$ post-injection.

Based on a slow exponential half-time of $40 \mathrm{~min}$, it can be seen from Fig. 4 that total tissue concentration (FDG6P + FDG) increases or decreases as a function of post-injection time for values of $\mathrm{Ki} / V(0)$ respectively greater than or less than $\beta(0.0173 \mathrm{ml} / \mathrm{min} / \mathrm{ml})$. For slow exponential half-times of 50 and $60 \mathrm{~min}, \beta$ is 0.0139

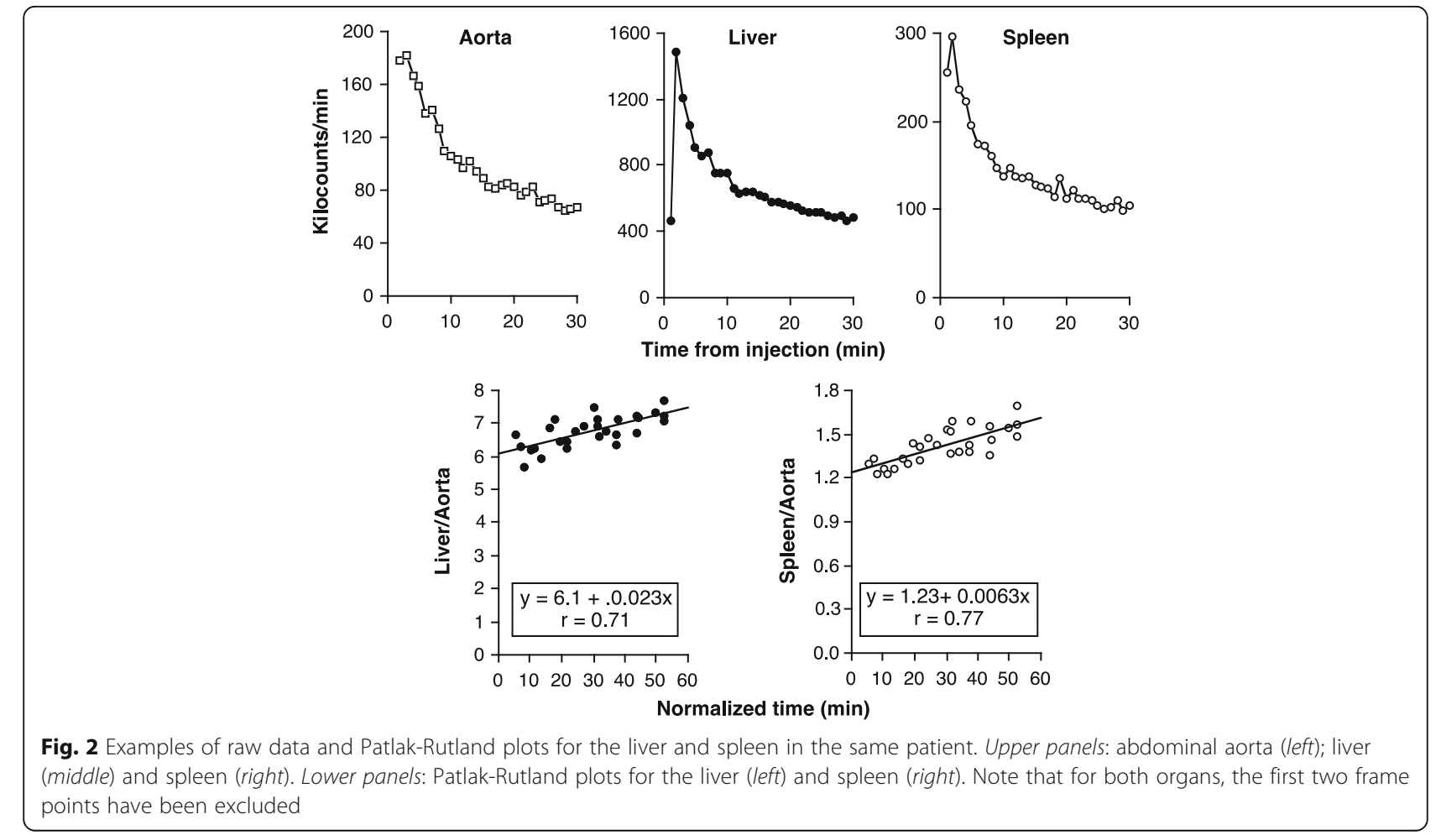




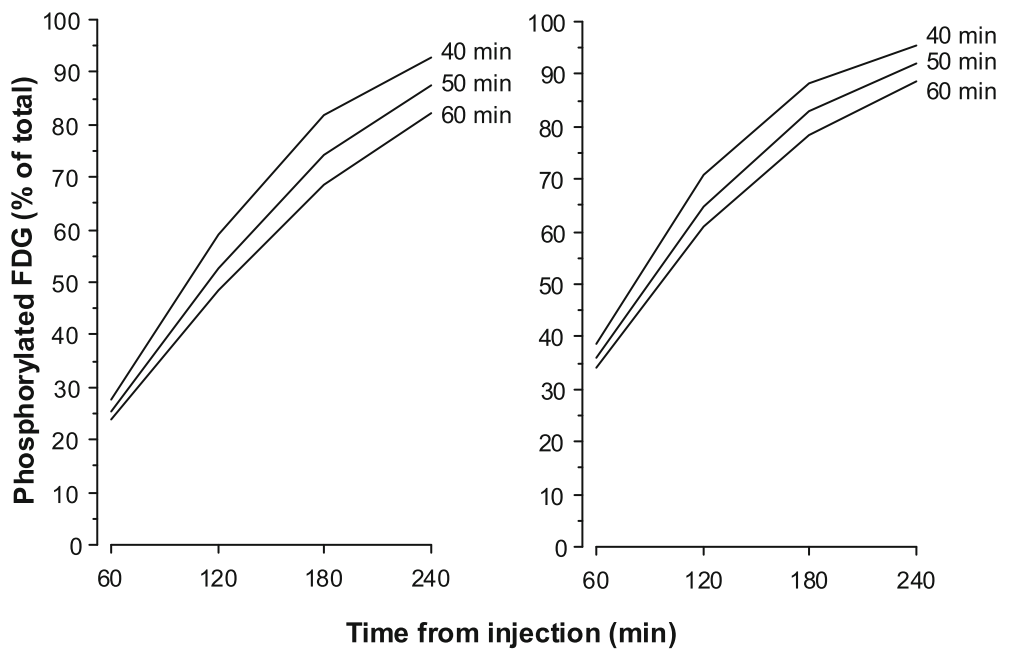

Fig. 3 Percentage of tissue FDG that is phosphorylated as a function of time after FDG injection shown for slow exponential half-times of 40, 50 and $60 \mathrm{~min}$; left panel: $\mathrm{Ki} / \mathrm{V}(0)=0.0036 \mathrm{ml} / \mathrm{min} / \mathrm{ml}$ (typical for the liver); right panel: $\mathrm{Ki} / \mathrm{V}(0)=0.006 \mathrm{ml} / \mathrm{min} / \mathrm{ml}$ (typical for the spleen)

and $0.0115 \mathrm{~min}^{-1}$, so the boundary $\mathrm{Ki} / V(0)$ values are 0.0139 and $0.0115 \mathrm{ml} / \mathrm{min} / \mathrm{ml}$, respectively.

\section{SUV indices at 60 min post-injection}

Hepatic SUV was higher than splenic SUV. Thus, the liver-to-spleen $\mathrm{SUV}_{\text {mean }}$ and $\mathrm{SUV}_{\max }$ ratios (which, as ratios, are independent of whole-body metric) were 1.23 $(0.24)$ and $1.36(0.26)$, respectively $(p<0.0001$ versus 1 for both). SUV indices generally correlated weakly with $\mathrm{Ki} / V(0)$ with respect to both the liver and spleen
(Table 1). With respect to the liver, but not the spleen, SUL indices correlated more strongly with $\mathrm{Ki} / V(0)$ than SUW indices, and $\mathrm{SUV}_{\max }$ indices correlated more strongly than $\mathrm{SUV}_{\text {mean }}$ indices. Adjusting the liver $\mathrm{SUV}_{\text {mean }}$ indices for hepatic fat improved their correlations with $\mathrm{Ki} /$ $V(0)$. Relationships of SUV indices with $\mathrm{Ki} / \mathrm{V}(0)$ had relatively large intercepts (Fig. 5), which, because SUV reflects the total tissue concentration, is consistent with Eq. 9. With respect to the spleen, division of SUV indices by hepatic $\mathrm{SUV}_{\text {mean }}$ improved their correlations

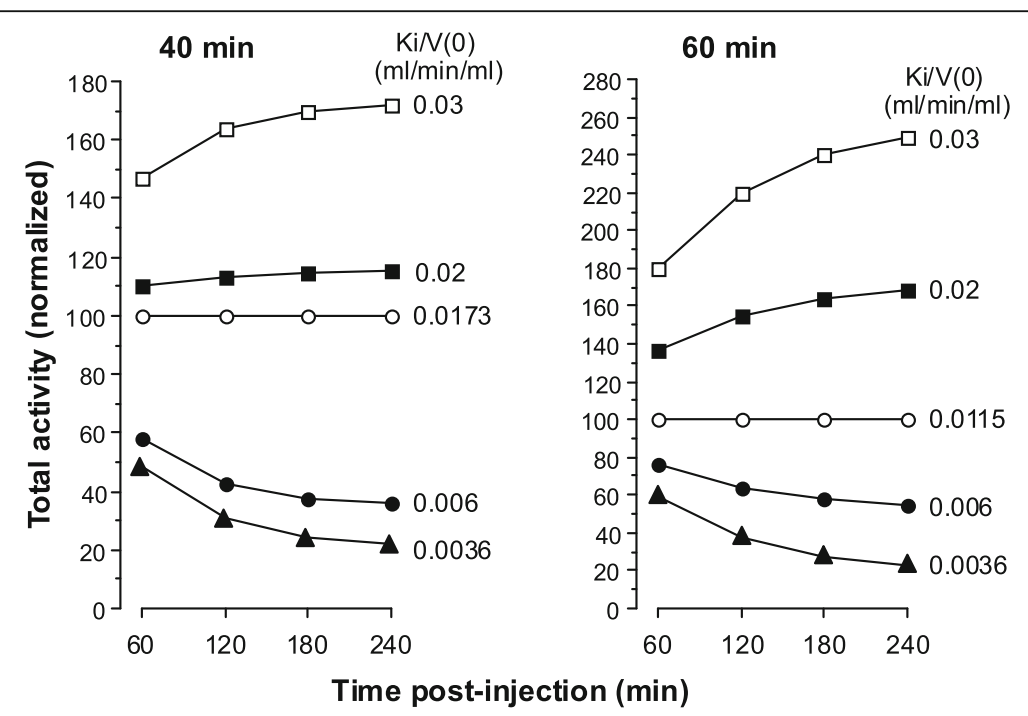

Fig. 4 Time courses of total tissue activity estimated using Eq. 9 for slow exponential half-times of 40 min (left panel) and 60 min (right panel). V0 has been set to unity. The vertical axis is the total tissue activity (FDG6P + FDG) normalized to 100. When $t$ is infinity, $C_{t}=0$ and $A_{t}=N / \beta$ (Eqs. 5 and 6 ). Therefore, when $\mathrm{Ki} / \mathrm{V}(0)=\beta$, the total activity is constant over post-injection time. For a slow exponential half-time of $40 \mathrm{~min}, \beta=0.0173 \mathrm{~min}{ }^{-1}$. Therefore, for values of $\mathrm{Ki} / \mathrm{V}(0)>0.0173 \mathrm{ml} / \mathrm{min} / \mathrm{ml}$, total activity increases as a function of post-injection time, and vice versa when $\mathrm{Ki} / \mathrm{V}(0)<0.0173 \mathrm{ml} / \mathrm{min} /$ $\mathrm{ml}$. The boundary $\mathrm{Ki} / V(0)$ value for a slow exponential half-time of $60 \mathrm{~min}$ is $0.0115 \mathrm{ml} / \mathrm{min} / \mathrm{ml}$. Ignoring the small areas under the fast exponentials does not alter these time courses 
Table 1 Correlation coefficients ( $p$ ) of relationships of hepatic and splenic SUV indices with hepatic and splenic Ki/V(0)

\begin{tabular}{lll}
\hline & Liver & Spleen \\
\hline SUW & $0.17(0.36)$ & $0.24(0.065)$ \\
SUW $_{\text {max }}$ & $0.29(0.045)$ & $0.24(0.065)$ \\
SUL $_{\text {mean }}$ & $0.20(0.17)$ & $0.25(0.054)$ \\
SUL & $0.38(0.005)$ & $0.26(0.045)$ \\
FA SUW & $0.31(0.030)$ & - \\
FA SUL & $0.40(0.0015)$ & - \\
SUV & & $0.40(0.0015)$ \\
SUV $_{\text {max }} /$ /hepatic & SUV & \\
\hline
\end{tabular}

with $\mathrm{Ki} / \mathrm{V}(0)$ but no differences were seen between $\mathrm{SUV}_{\text {max }}$ and $\mathrm{SUV}_{\text {mean }}$ indices.

\section{Discussion}

We found that at 60 min post-injection, only about $25 \%$ of FDG is phosphorylated in tissues, such as the liver, with $\mathrm{Ki} / \mathrm{V}(0)$ of $0.0036 \mathrm{ml} / \mathrm{min} / \mathrm{ml}$, and about $35 \%$ in tissues, such as the spleen, with $\mathrm{Ki} / \mathrm{V}(0)$ of $0.006 \mathrm{ml} / \mathrm{min} / \mathrm{ml}$. The half-time of the assumed exponential decrease in blood FDG concentration (40,50 or $60 \mathrm{~min}$ ) had little influence on these percentages, although, as would be expected, the percentages were slightly higher for faster half-times. The remaining FDG is un-phosphorylated, exchanging with blood FDG. These percentages increased at later times post-injection but did not approach $100 \%$ until several hours post-injection.

Whilst there has been much interest in measuring FDG clearance in several tissues for many years, there

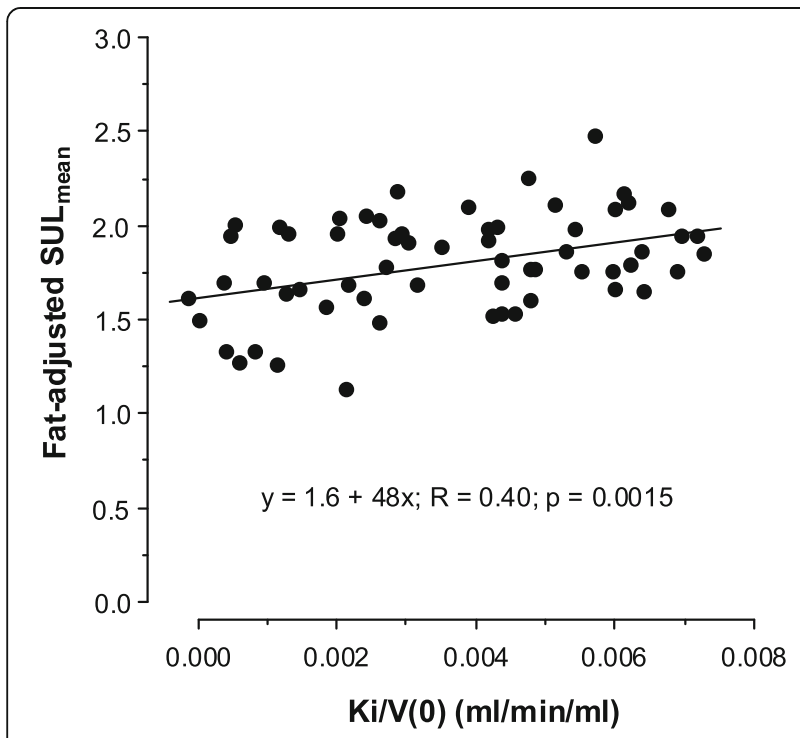

Fig. 5 Relationship between fat-adjusted hepatic $S U L_{\text {mean }}$ and $\mathrm{Ki} / V(0)$ (see Table 1). Note the large positive zero time intercept, consistent with Eq. 9 has been less interest in the physiological significance of tissue FDG distribution volume. Yet, as shown here, $V(0)$ is critical in determining the proportion of FDG in a tissue that is actually phosphorylated. Several studies have reported how the SUVs of various tissues change between imaging at $60 \mathrm{~min}$ and delayed imaging, with some tissues showing an increase and others, notably the liver and spleen, showing a decrease in SUV [33-37]. The magnitudes and directions of the changes are determined by $\mathrm{Ki} / V(0)$. Thus, when $\mathrm{Ki} / V(0)$ is higher than $\beta$, there is an increase in SUV and vice versa when $\mathrm{Ki} / V(0)$ is lower than $\beta$. The issue of early versus delayed imaging is likely to increase in importance with the development of new whole-body PET machines such as UltraPET [38].

In the measurement, by computer modelling, of the transport constants in Fig. 1, previous workers have stressed the importance of considering the liver's dual blood supply [1-3, 39]. There is general agreement, however, that Patlak-Rutland analysis, although less informative, is not influenced by the dual blood supply and is more robust than computer modelling for the measurement of $\mathrm{Ki}$ and $V(0)$. Table 2 summarizes values of Ki and $V(0)$ obtained by previous workers using computer modelling and Patlak-Rutland analysis. It can be seen from this Table that in humans, there is good agreement between modelling and Patlak-Rutland analysis [2] and that our mean value of $\mathrm{Ki} / V(0)$ for the liver, based on Patlak-Rutland analysis, is similar to previously published values in humans based on both modelling and Patlak-Rutland analysis [1, 2]. In anaesthetised pigs, Ki based on modelling was somewhat higher than human values [3]. Patlak-Rutland analysis, however, gave values of $\mathrm{Ki} / V(0)$ in line with other studies and our own values [1-3]. We can conclude therefore that our estimate of $\mathrm{Ki} / V(0)$ for the liver is reliable. We could find no corresponding literature values for the spleen.

Table 2 Hepatic Ki and V(0) determined by previous workers [1-3] using computer modelling and Patlak-Rutland graphical analysis

\begin{tabular}{lllllllll}
\hline & \multicolumn{3}{l}{ Modelling } & & \multicolumn{3}{c}{ Graphical analysis } \\
\cline { 2 - 3 } & Ki & $V(0)$ & $\mathrm{Ki} / V(0)$ & & $\mathrm{Ki}$ & $V(0)$ & $\mathrm{Ki} / V(0)$ \\
\hline Choi et al. [1] & 0.0043 & $0.88^{\mathrm{a}}$ & 0.0049 & & $\mathrm{ND}$ & $\mathrm{ND}$ & $\mathrm{ND}$ \\
lozzo et al. [2] & 0.0029 & 0.81 & 0.0036 & & 0.0023 & $0.84^{\mathrm{b}}$ & $0.0029^{\mathrm{b}}$ \\
Munk et al. [3] $^{c}$ & 0.0078 & 0.95 & 0.0082 & & 0.0037 & 1.05 & 0.0035
\end{tabular}

$\mathrm{Ki}$ and $V(0)$ based on modelling have been calculated using the equations linking $\mathrm{Ki}$ and $V(0)$ to the transport constants (e.g. $\mathrm{Ki}=K_{1} \times k_{3} /\left[k_{2}+k_{3}\right]$ and $\left.V(0)=K_{1} /\left[k_{2}+k_{3}\right]\right)$

$\mathrm{Ki}, \mathrm{ml} / \mathrm{min} / \mathrm{ml} ; V(0), \mathrm{ml} / \mathrm{ml}$. Note that our mean value of $\mathrm{Ki} / \mathrm{V}(0)$ was $0.0036 \mathrm{ml} / \mathrm{min} / \mathrm{ml}$

ND not done

FA - fat-adjusted

${ }^{\mathrm{a}} \mathrm{ml} / \mathrm{g}$

${ }^{\text {b}}$ Values from single illustrated Patlak-Rutland plot (mean of group values not given) ${ }^{\mathrm{C}}$ Anaesthetized pigs 
The spleen SUV was found to be less than the liver SUV even though it had a higher $\mathrm{Ki} / V(0)$. As shown in the Appendix, this is the result of a lower distribution volume in the spleen. Considering the relative values of hepatic and splenic $\mathrm{SUV}_{\max }$ and $\mathrm{Ki} / V(0)$, hepatic $V(0)$ can be calculated to be 1.6-fold higher than splenic $V(0)$ (see Appendix).

SUV depends on the total tissue FDG concentration (FDG plus FDG6P), which partly explains the weak correlations we found between SUV indices and $\mathrm{Ki} / V(0)$ for both the liver and spleen. Interestingly, the strongest correlations were found with SUL indices, which support the use of LBM for calculating SUV [40]. The poor correlations of hepatic $\mathrm{SUV}_{\text {mean }}$ indices with $\mathrm{Ki} / V(0)$ is explained by the physical effect of hepatic fat on the FDG signal-a 'fat-diluting' effect that has previously been described to affect $S U V_{\text {max }}$ less than $S_{U V}$ mean [32]. Making the described adjustment for hepatic fat gave $\mathrm{SUV}_{\text {mean }}$ a stronger correlation with $\mathrm{Ki} / V(0)$ compared with the corresponding unadjusted values (approximately doubling the correlation coefficients for both $\mathrm{SUW}_{\text {mean }}$ and $\mathrm{SUL}_{\text {mean; }}$ Table 1). With respect to the spleen, correlations were similar for $\mathrm{SUV}_{\text {max }}$ and $\mathrm{SUV}_{\text {mean }}$ indices because fat is not an issue in the spleen. Interestingly, the strongest correlation with $\mathrm{Ki} / V(0)$ was seen when splenic SUV was divided by hepatic SUV. Others have previously shown that division of tissue SUV by blood pool SUV makes it a better surrogate of clearance than SUV alone $[41,42]$. As shown here, hepatic SUV is largely a blood pool SUV, explaining this finding.

A potential limitation of Patlak-Rutland analysis is the influence of glucose-6-phosphatase $\left(k_{4}\right)$, which if active would invalidate the requirement for a single transport pathway. Previous workers in this field, however, also recorded linear hepatic Patlak-Rutkand gradients, suggesting that de-phosphorylation is very slow [1-3]. In any event, we avoided a longer acquisition period in order to minimize a possible influence of de-phosphorylation on the gradient.

Study limitations include firstly the recruitment of patients with co-existing morbidity. Secondly, hepatic fat distribution is heterogeneous [43], so measurement of CT density in a single ROI for the hepatic fat adjustment procedure may be misleading. Moreover, CT is not regarded as the gold standard imaging technique for quantifying steatosis, but probably instead MRI and MR spectroscopy [44]. The duration of our dynamic acquisition may be considered limited but longer acquisition periods risk patient movement artefacts, especially in patient participants rather than motivated normal volunteers $[1,2,4]$, as well as a possible influence of dephosphorylation, so $30 \mathrm{~min}$ seemed reasonable. Others used $40[2,4]$ or $60 \mathrm{~min}$ [1]. A 2-min mixing time of FDG throughout the hepatic distribution volume may seem brief but is consistent with previously reported values of $K_{1}$ and $k_{2}$, which respectively ranged from 0.01 to 0.015 and 0.013 to $0.016 \mathrm{~s}^{-1}[1-3]$, and which therefore give an equilibration rate constant of $0.023-0.031 \mathrm{~s}^{-1}$. This gives a time to $95 \%$ equilibration of FDG between compartments 1 and 2 of 97-130 s. Munk et al. also found from rapid early sampling that equilibration was achieved within a few minutes [3]. We used abdominal aorta for arterial input but others have validated the abdominal aorta for Patlak-Rutland analysis [28, 45], including for the liver [45].

\section{Conclusions}

In conclusion, tissue FDG6P concentration depends on FDG clearance per unit total volume but the FDG6P/ FDG concentration ratio depends on tissue clearance per unit distribution volume. Because the majority of FDG is un-phosphorylated, SUV in both the liver and spleen largely reflects blood pool activity, especially the liver, explaining the poor correlations between SUV indices and $\mathrm{Ki} / V(0)$. If $\mathrm{SUV}$ is to be used as a measure of FDG clearance and metabolic activity, then LBM is the preferred whole-body metric with which to calculate it.

\section{Appendix}

Equation 9 can be re-written, replacing FDG6 + FDG with SUV, for both liver (L) and spleen (S).

$$
\frac{\mathrm{SUV}_{S}}{\mathrm{SUV}_{L}}=\frac{\left(V(0) \cdot A(t) \cdot[\mathrm{Ki} / V(0)]_{S}\right)+V(0)_{S} \cdot C(t)}{\left(V(0) \cdot A(t) \cdot[\mathrm{Ki} / V(0)]_{L}\right)+V(0)_{L} \cdot C(t)}
$$

Note that administered activity and whole-body metric cancel out when the two SUVs are expressed as a ratio, which then becomes the ratio of the total FDG concentrations in the two organs.

Therefore, re-arranging and dividing numerator and denominator in Eq. A1 by $C(t)$

$$
\frac{\mathrm{V}(0)_{L}}{\mathrm{~V}(0)_{S}}=\frac{\left.\mathrm{SUV}_{L} \times[A(t) / C(t) \cdot][\mathrm{Ki} / V(0)]_{S}+1\right)}{\left.\mathrm{SUV}_{S} \times[A(t) / C(t)] \cdot[\mathrm{Ki} / V(0)]_{L}+1\right)}
$$

For disappearance exponential half-times of 40, 50 and $60 \mathrm{~min}, A(t) / C(t)$ is 105,94 and $87 \mathrm{~min}$, respectively. So for $\mathrm{SUV}_{\mathrm{L}} / \mathrm{SUV}_{\mathrm{S}}$ of 1.36 (see the "Results" section), the corresponding values of $V(0)_{\mathrm{L}} / V(0)_{\mathrm{S}}$ would be $1.61,1.59$ and 1.58 .

\section{Funding}

This study was not funded.

Authors' contributions

GK designed the study, consented the patients, acquired the data and analysed the data. CDA co-wrote the paper. AMP obtained the ethical approval, designed 
the study, analysed the data and co-wrote paper. All authors read and approved the final manuscript.

\section{Competing interests}

The authors declare that they have no competing interests.

\section{Ethics approval and consent to participate}

All procedures performed in studies involving human participants were in accordance with the ethical standards of the institutional and/or national research committee and with the 1964 Helsinki declaration and its later amendments or comparable ethical standards. All patients gave written informed consent for the study, which was approved by a local institutional review board (NRES Committee South Central - Oxford C: ref 13/SC/0231). No animals were involved in this study.

\section{Author details}

${ }^{1}$ Clinical Imaging Sciences Centre, Brighton Sussex Medical School, Brighton, UK. ${ }^{2}$ Center for Experimental Surgery, Clinical and Translational Research, Biomedical Research Foundation Academy of Athens, Athens, Greece. ${ }^{3}$ Department of Nuclear Medicine, Royal Sussex County Hospital, Eastern Road, Brighton BN2 5BE, UK.

\section{Received: 7 December 2016 Accepted: 4 January 2017} Published online: 07 February 2017

\section{References}

1. Choi Y, Hawkins RA, Huang SC, et al. Evaluation of the effect of glucose ingestion and kinetic model configurations of FDG in the normal liver. J Nucl Med. 1994;35:818-23.

2. Iozzo P, Geisler F, Oikonen V, et al. Insulin stimulates liver glucose uptake in humans: an ${ }^{18}$ F-FDG PET Study. J Nucl Med. 2003;44:682-9.

3. Munk OL, Bass L, Roelsgaard K, Bender D, Hansen SB, Keiding S. Liver kinetics of glucose analogs measured in pigs by PET: importance of dualinput blood sampling. J Nucl Med. 2001;42:795-801.

4. Borra R, Lautamaki R, Parkkola R, et al. Inverse association between liver fat content and hepatic glucose uptake in patients with type 2 diabetes mellitus. Metabolism. 2008;57:1445-51.

5. Lindholm P, Minn H, Leskinen-Kallio S, et al. Influence of the blood glucose concentration on FDG uptake in cancer-a PET study. J Nucl Med. 1993;34:1-6.

6. Namba $H$, Nakagawa $K$, lyo $M$, et al. A simple method for measuring glucose utilization of insulin-sensitive tissues by using the brain as a reference. Eur J Nucl Med. 1994;21:228-31.

7. Hasselbalch SG, Knudsen GM, Capaldo B, et al. Blood-brain barrier transport and brain metabolism of glucose during acute hyperglycemia in humans. J Clin Endocrinol Metab. 2001;86:1986-90.

8. Boellaard R, O'Doherty MJ, Weber WA, et al. FDG PET and PET/CT: EANM procedure guidelines for tumour PET imaging: version 1.0. Eur J Nucl Med Mol Imaging. 2010;37:181-200.

9. Abikhzer G, Alabed YZ, Azoulay L, Assayag J, Rush C. Altered hepatic metabolic activity in patients with hepatic steatosis on FDGPET/CT. Am J Roentgenol. 2011;196:176-80.

10. Abele JT, Fung Cl. Effect of hepatic steatosis on liver FDG uptake measured in mean standard uptake values. Radiology. 2010;254:917-24.

11. Lin CY, Lin WY, Lin CC, et al. The negative impact of fatty liver on maximum standard uptake value of liver on FDG PET. Clin Imaging. 2011;35:437-41.

12. Kumar R, Xiu Y, Yu JQ, et al. ${ }^{18} \mathrm{~F}$-FDG PET in evaluation of adrenal lesions in patients with lung cancer. J Nucl Med. 2004;45:2058-62.

13. Bural GG, Torigian DA, Burke A, et al. Quantitative assessment of the hepatic metabolic volume product in patients with diffuse hepatic steatosis and normal controls through use of FDG-PET and MR imaging: a novel concept. Mol Imaging Biol. 2010;12:233-9.

14. Kim EJ, Kim S, Kang DO, et al. Metabolic activity of the spleen and bone marrow in patients with acute myocardial infarction evaluated by ${ }^{18} \mathrm{~F}$-fluorodeoxyglucose positron emission tomographic imaging. Circ Cardiovasc Imaging. 2014;7:454-60.

15. Emami $H$, Singh $P$, MacNabb M, et al. Splenic metabolic activity predicts risk of future cardiovascular events: demonstration of a cardiosplenic axis in humans. JACC Cardiovasc Imaging. 2015;8:121-30.

16. Wollenweber $T$, Roentgen $P$, Schafer $A$, et al. Characterizing the inflammatory tissue response to acute myocardial infarction by clinical multimodality noninvasive imaging. Circ Cardiovasc Imaging. 2014;7:811-8.
17. Boellaard R, van Lingen A, Lammertsma AA. Experimental and clinical evaluation of iterative reconstruction (OSEM) in dynamic PET: quantitative characteristics and effects on kinetic modeling. J Nucl Med. 2001;42:808-17.

18. Wakita $K$, Imahori $Y$, Ido $T$, et al. Simplification for measuring input function of FDG PET: investigation of 1-point blood sampling method. J Nucl Med. 2000:41:1484-90.

19. Shiozaki T, Sadato N, Senda M, et al. Noninvasive estimation of FDG input function for quantification of cerebral metabolic rate of glucose: optimization and multicenter evaluation. J Nucl Med. 2000;41:1612-8.

20. Tsuchida T, Sadato N, Yonekura $Y$, et al. Noninvasive measurement of cerebral metabolic rate of glucose using standardized input function. J Nucl Med. 1999:40:1441-5.

21. Huang J, O'Sullivan F. An analysis of whole body tracer kinetics in dynamic PET studies with application to image-based blood input function extraction. IEEE Trans Med Imaging. 2014;33:1093-108.

22. Zhou S, Chen K, Reiman EM, Li DM, Shan B. A method of generating imagederived input function in a quantitative ${ }^{18}$ F-FDG PET study based on the shape of the input function curve. Nucl Med Commun. 2011;32:1121-7.

23. Vriens D, de Geus-Oei L-F, Oyen WJG, Visser EP. A curve-fitting approach to estimate the arterial plasma input function for the assessment of glucose metabolic rate and response to treatment. J Nucl Med. 2009:50:1933-9.

24. O'Sullivan F, Muzi M, Spence AM, et al. Nonparametric residue analysis of dynamic PET data with application to cerebral FDG studies in normals. J Am Stat Assoc. 2009;104(486):556-71.

25. O'Sullivan F, Kirrane J, Muzi M, et al. Kinetic quantitation of cerebral PET-FDG studies without concurrent blood sampling: statistical recovery of the arterial input function. IEEE Trans Med Imaging. 2010;29:610-24.

26. Guo H, Renaut RA, Chen K. An input function estimation method for FDG PET human brain studies. Nucl Med Biol. 2007;34:483-92.

27. van der Weerdt AP, Klein $\sqcup$, Boellaard R, Visser CA, Visser FC, Lammertsma AA. Image-derived input functions for determination of MRGlu in cardiac (18)FFDG PET scans. J Nucl Med. 2001;42:1622-9.

28. de Geus-Oei LF, Visser EP, Krabbe PF, van Hoorn BA, Koenders EB, Willemsen AT, et al. Comparison of image-derived and arterial input functions for estimating the rate of glucose metabolism in therapy-monitoring ${ }^{18}$ F-FDG PET studies. J Nucl Med. 2006;47:945-9.

29. Keramida G, Hunter J, Peters AM. Hepatic glucose utilisation in hepatic steatosis and obesity. Biosci Rep. 2016;36: article Identifier: e00402 DOl*: doi:10.1042/BSR20160381.

30. Boer $P$. Estimated lean body mass as an index for normalization of body fluid volumes in man. Am J Physiol. 1984;247:F632-5.

31. Ricci $C$, Longo $R$, Gioulis $E$, et al. Noninvasive in vivo quantitative assessment of fat content in human liver. J Hepatol. 1997;27:108-13.

32. Keramida G, Potts J, Bush J, Verma S, Dizdarevic, Peters AM. Accumulation of ${ }^{18} \mathrm{~F}-\mathrm{FDG}$ in the liver in hepatic steatosis. Am J Roentgenol. 2014;203:643-8.

33. Cheng G, Alavi A, Lim E, Werner TJ, Del Bello CV, Akers SR. Dynamic changes of FDG uptake and clearance in normal tissues. Mol Imaging Bio. 2013;15:345-52.

34. Lee JW, Kim SK, Lee SM, Moon SH, Kim TS. Detection of hepatic metastases using dual-time-point FDG PET/CT scans in patients with colorectal cancer. Mol Imaging Biol. 2011;13:565-72.

35. Basu S, Kung J, Houseni M, Zhuang H, Tidmarsh GF, Alavi A. Temporal profile of fluorodeoxyglucose uptake in malignant lesions and normal organs over extended time periods in patients with lung carcinoma: implications for its utilization in assessing malignant lesions. Q J Nucl Med Mol Imaging. 2009;53:9-19.

36. Chirindel A, Alluri KC, Tahari AK, et al. Liver standardized uptake value corrected for lean body mass at FDG PET/CT: effect of FDG uptake time. Clin Nucl Med. 2015;40:e17-22.

37. Mejia AA, Nakamura T, Masatoshi I, Hatazawa J, Masaki M, Watanuki S. Estimation of absorbed doses in humans due to intravenous administration of fluorine-18-fluorodeoxyglucose in PET studies. J Nucl Med. 1991;32:699-706.

38. Price PM, Badawi RD, Cherry SR, Jones T. Ultra staging to unmask the prescribing of adjuvant therapy in cancer patients: the future opportunity to image micrometastases using total-body ${ }^{18}$ F-FDG PET scanning. J Nucl Med. 2014;55:696-7.

39. Tragardh M, Moller N, Sorensen M. Methodologic considerations for quantitative ${ }^{18} \mathrm{~F}$-FDGPET/CT studies of hepatic glucose metabolismin healthy subjects. J Nucl Med. 2015;56:1366-71.

40. Sugawara Y, Zasadny KR, Neuhoff AW, Wahl RL. Reevaluation of the standardized uptake value for FDG: variations with body weight and methods for correction. Radiology. 1999;213:521-5. 
41. van den Hoff J, Oehme L, Schramm G, et al. The PET-derived tumor-toblood standard uptake ratio (SUR) is superior to tumor SUV as a surrogate parameter of the metabolic rate of FDG. EJNMMI Res. 2013;3(1):77.

42. Keramida G, Dizdarevic S, Bush J, Potts J, Peters AM. Quantification of tumour ${ }^{18} \mathrm{~F}-\mathrm{FDG}$ uptake: normalise to blood glucose or scale to liver uptake? Eur Radiol. 2015;25:2701-8.

43. Decarie PO, Lepanto L, Billiard JS, et al. Fatty liver deposition and sparing: a pictorial review. Insights Imaging. 2011;2:533-8.

44. Bohte AE, van Werven JR, Bipat S, Stoker J. The diagnostic accuracy of US, CT, MRI and ${ }^{1} \mathrm{H}-\mathrm{MRS}$ for the evaluation of hepatic steatosis compared with liver biopsy: a meta-analysis. Eur Radiol. 2011;21:87-97.

45. Keiding S, Munk OL, Schiøtt KM, Hansen SB. Dynamic 2-[ ${ }^{18}$ F]fluoro-2-deoxyD-glucose positron emission tomography of liver tumours without blood sampling. Eur J Nucl Med. 2000;27:407-12.

\section{Submit your manuscript to a SpringerOpen ${ }^{\circ}$ journal and benefit from:}

- Convenient online submission

- Rigorous peer review

- Immediate publication on acceptance

- Open access: articles freely available online

- High visibility within the field

- Retaining the copyright to your article

Submit your next manuscript at $\boldsymbol{s p r i n g e r o p e n . c o m ~}$ 